\title{
Quantitative Effect of Emergency Department Case Management on Visits, Diagnostics, and Cost
}

Jeffrey Nickel, MD ${ }^{1}$, Nancy Connelly ${ }^{1}$, Cameron Duffner ${ }^{2}$, Xyryl Pablo ${ }^{2}$, Aeleia Hughes $^{3}$

${ }^{1}$ Parkview Health, ${ }^{2}$ Indiana University School of Medicine, ${ }^{3}$ Baylor University

\section{Background and Hypothesis:}

In 2012, Parkview Health initiated a case management (CM) program with the hypothesis that it would reduce emergency department (ED) visits, radiation exposure, and costs for patients who had visited a Parkview ED 5 or more times within 6 months.

\section{Experimental Design or Project Methods:}

This retrospective case series involved examining medical records of 460 CM patients from 2011 to 2018, recording the amount of Parkview ED visits, diagnostic tests, and affected cost accumulated in the year prior to CM enrollment compared to each of the next 2 years. Demographics, chief complaints, diagnoses, psychiatric and drug use history, and whether the patients had insurance and a primary care provider were also recorded. Patient data was excluded if the patient was younger than 18 at the time of $\mathrm{CM}$ enrollment, had not yet completed 2 years in the CM program, or if medical records were not available. ANOVA and 1-sided, paired t-testing were performed to evaluate significance of the results.

\section{Results:}

Comparing the year before enrollment to the $2^{\text {nd }}$ year after, ED visits were reduced from 5,264 to 2,012 for 378 patients $(63 \%, p<0.01)$, the affected cost was reduced from $\$ 551,734.45$ to $\$ 246,248.34$ for 299 patients $(55 \%, p<0.01)$, and the number of diagnostic tests was reduced from 6,040 to 1,883 for 104 patients $(69 \%, p<0.01)$.

\section{Conclusion and Potential Impact:}

Patients enrolled in Parkview's CM program showed statistically significant reductions in ED visits, radiologic exposures, and affected costs over 2 years, with implicit improved health outcomes. Projected 10-year affected cost savings range from $\$ 3.7$ million to $\$ 9.1$ million. 